\title{
Pattern of Adverse Transfusion Reactions in Acute Leukaemia Treated with Different Types of Blood Component Therapy in a Tertiary Care Hospital
}

\author{
*Yamani MJ1, Hossain $\mathrm{MG}^{2}$, Chowdhury MAH${ }^{3}$, Hossain $\mathrm{MI}^{4}$, Hossain $\mathrm{MM}^{5}$, Khan $\mathrm{MR}^{6}$, Shah MS ${ }^{7}$, \\ Aziz $\mathrm{MA}^{8}$, Yunus $\mathrm{ABM}^{9}$
}

\begin{abstract}
Background: Transfusion of blood products is one of the principle components of supportive management in patients with acute leukaemia. Objective: The purpose of this study was to observe the pattern of adverse transfusion reactions (ATR) in acute leukaemia patients receiving blood component therapy. Methodology: This observational study was conducted in the Department of Haematology, Bangabandhu Sheikh Mujib Medical University (BSMMU), Dhaka, Bangladesh from January 2018 to December 2018. Total ninety-five diagnosed case of Acute Myeloid Leukaemia (AML) and Acute Lymphoblastic Leukaemia (ALL) patients were selected for the study. Results: Four types of transfusion reactions including Febrile Non-Haemolytic Transfusion Reaction (FNHTR), Allergic, Anaphylactic, Delayed Haemolytic Transfusion Reaction (DHTR) were detected by clinical observations and relevant laboratory investigations. In this study, 25 (26.3\%) patients showed different types of adverse transfusion reactions. Allergic reactions (48\%) found to be the most common followed by FNHTR (32\%), anaphylactic reactions (16\%) and DHTR (4\%). Allergic reactions $(58.34 \%)$ were predominant in platelet transfusion and febrile reactions (62.5\%) observed in red cell concentrate (RCC) transfusion. Urticaria, pruritus, angioedema, breathlessness, stridor, shivering, hypotension were prominent symptoms in allergic and anaphylactic reactions. On the other hand, fever, chills and rigors were prominent symptoms in case of febrile non haemolytic transfusion reaction. In our study no association between transfusion reaction with age, sex, types of donor, types of platelet and unit of transfusion was found. ATRs are mostly non-severe but rarely cause severe transfusion reaction. Conclusion: For safe blood transfusion close monitoring of transfusion, early recognition of pattern of reaction and prompt action may decrease transfusion related adverse events.
\end{abstract}

Key words: Acute leukaemia, blood component therapy, adverse transfusion reactions.

\section{Author's}

1. *Md. Jaki Yamani, Department of Haematology, National Institute of Cancer Research and Hospital, Dhaka, Bangladesh.

2. Md. Golzar Hossain, Department of Haematology, National Institute of Cancer Research and Hospital, Dhaka, Bangladesh.

3. Md. Ashraful Haque Chowdhury, Department of haematology and BMT, Dhaka Medical College, Dhaka, Bangladesh.

4. Md. Imran Hossain, Department of Haematology, Bangabandhu Sheikh Mujib Medical University (BSMMU), Shahbag, Dhaka, Bangladesh.

5. Mohammed Murad Hossain, Department of Haematology, National Institute of Cancer Research and Hospital, Dhaka, Bangladesh.

6. Md. Rafiquzzaman Khan, Department of Haematology, Bangabandhu Sheikh Mujib Medical University, Dhaka, Bangladesh.

7. Md. Salahuddin Shah, Department of Haematology, Bangabandhu Sheikh Mujib Medical University, Dhaka, Bangladesh.

8. Md. Abdul Aziz, Department of Haematology, Bangabandhu Sheikh Mujib Medical University, Dhaka, Bangladesh.

9. ABM Yunus, Department of Haematology, Bangabandhu Sheikh Mujib Medical University, Dhaka, Bangladesh.

Correspondence: Md. Jaki Yamani, Department of Haematology, Bangabandhu Sheikh Mujib Medical University, Dhaka, Bangladesh, e-mail:jakiabir@yahoo.com,Contact:+880 1673-020127.

Page | 35

\section{Introduction}

Acute leukaemia including Acute Myeloid Leukaemia (AML) and Acute Lymphoblastic Leukaemia (ALL) are the most prevalent Haematological Malignancy (HM) affecting Bangladeshi population, accounting for $42.4 \%$ of all HM cases, while these two constituted $66 \%$ of leukaemia cases. ${ }^{1}$ The overall incidence of AML is 3.7 cases per 100,000 of the population. The median age at diagnosis is approximately 70 years and $66 \%$ is over 60 years of age. ${ }^{2}$ ALL is the most common malignancy in childhood with the majority of cases in the 2-10 years of age group (median 3.5 years) but rare leukaemia in adults, 0.7-1.8/100000 annually. In adults, peak at 15-24 years with further peak in older age (2.3/100000 among $>80$ years). ${ }^{3}$ In the management of acute leukaemia requires cytotoxic chemotherapy, prolonged hospitalization and aggressive 
supportive care including transfusion of blood products (red cell cone, platelet cone, Fresh Frozen Plasma).

Despite advances in modern blood banking procedures and all the precaution taken, a small but significant number of blood transfusions are still complicated by various kinds of adverse reactions. Adverse transfusion reactions (ATR) are mostly not severe but rarely cause severe diseases including transfusion related acute lung injury (TRALI), anaphylactic shock and acute haemolytic transfusion reaction (AHTR).

Acute leukaemia patient receiving immunosuppressive drug in chemotherapy schedule which can musk transfusion reaction so these patients needs special attention for diagnosis of transfusion reaction. Moreover, transfusion reaction pattern and frequency may vary significantly according to number of different variables. So, this study aimed to early detection of transfusion reaction and its pattern in acute leukaemia patient and which will help to generate better and effective management plan in order to contribute to safe blood transfusion and enhance life expectancy of leukaemia patient.

\section{Materials and Methods}

This observational study was conducted in the Department of Haematology, Bangabandhu Sheikh Mujib Medical University (BSMMU) from January 2018 to December 2018. Total ninety-five diagnosed AML and ALL patients were enrolled who met all inclusion criteria. The study was approved by the Institutional Review Board (IRB) of BSMMU. Only diagnosed case of AML and ALL $\geq 12$ years receiving or in need of transfusion during management were included in this study. Written consent was obtained from the acute leukaemia patients or from their guardians. Patients suffering from febrile illness and refused to give informed written consent were excluded from the study. Patient were interviewed, clinically examined and investigated for different types of adverse transfusion reactions if required. Clinical and laboratory information were recorded with a semi-structured data collection sheet. Categorical data was grouped as percent and numbers and mean with standard deviation measured from continuous data. Chi-square test was used to extract p-value. The statistical analysis was carried out using Statistical Package for Social Sciences (SPSS) version 24.0 for windows.

Haematol J Bangladesh 2019; 3(2):35-38

\section{Results}

Original Article

Among total patient $67.4 \%$ belongs to AML and $32.6 \%$ belongs to ALL group. Mean age for AML and ALL was $34 \pm 15$ years and $30 \pm 14$ years respectively. Female sex was predominance $(53.1 \%)$ in AML and male sex was predominance $(67.7 \%)$ in ALL. We did not find any significant relationship between age and sex with transfusion reaction. $31.37 \%$ male and $20.45 \%$ female subjects showed transfusion reaction (p: 0.228). Maximum reactions (36\%) were observed in 12- 21 years age group (P-value is 0.600$)$. Out of 95 subjects receiving blood component, red cell concentrate (RCC) was highest $48.4 \%$, Fresh Frozen Plasma (FFP) was lowest $11.6 \%$ and platelet concentrate having second $40 \%$. During study period $7.4 \%$ blood components were collected from blood relatives and 92.6\% from unrelated donor. Among them 14\% patient developed transfusion reaction with related donor and $27 \%$ with unrelated donor (p:0.453). In platelet cone transfusion maximum subjects $(65 \%)$ transfused random donor platelet while $35 \%$ subjects transfused single donor aphaeretic platelet. Frequency of transfusion reactions in aphaeretic platelet and random donor platelet transfusion was $34.61 \%$ and $42.85 \%$ respectively (p: 0.608$)$.

Table 1: Pattern of transfusion reaction

\begin{tabular}{lc}
\hline Pattern of reaction & $\mathbf{n}(\boldsymbol{\%})$ \\
\hline FNHTR & $8(32.0)$ \\
Allergic & $12(48.0)$ \\
Anaphylactic & $4(16.0)$ \\
DHTR & $1(4.0)$ \\
\hline
\end{tabular}

Among the $(26.3 \%)$ transfusion reactions $8(32 \%)$ were Febrile Non-Haemolytic Transfusion Reaction (FNHTR), 12 (48\%) were allergic reactions, 4(16\%) were anaphylactic reactions and 1(4\%) were Delayed Haemolytic Transfusion Reaction (DHTR). (Table 1) Male subjects were predominant $(75 \%)$ in allergic reaction but equal sex distribution (50\% male \& $50 \%$ female) in case of febrile and anaphylactic reaction.

Table 2: Distribution of transfusion reaction in different blood component

\begin{tabular}{lcccc}
\hline Types of reaction & RCC (\%) & Platelet conc. (\%) & FFP (\%) & P-value \\
\hline FNHTR & 62.5 & 37.5 & 0.0 & $0.208 \mathrm{~ns}$ \\
Allergic & 33.33 & 50.0 & 16.67 & \\
Anaphylactic & 0.0 & 100 & 0.0 & \\
\hline
\end{tabular}

The frequency of FNHTRs to RCC $(62.5 \%)$ was significantly higher than those to platelet concentrate $(37.5 \%)$. In contrast, the frequency of allergic Page | 36 
Original Article

reactions to platelet components $(50 \%)$ was significantly higher than RCC (33.33\%) and FFP (16.67\%). However, about $100 \%$ anaphylactic reactions were observed in case of platelet transfusion. (Table 2)

Table 3: Symptoms of transfusion reaction

\begin{tabular}{lc}
\hline Symptoms & $\mathbf{n}(\boldsymbol{\%})$ \\
\hline Fever & $8(16.3)$ \\
Urticaria & $12(24.5)$ \\
Breathlessness & $4(8.2)$ \\
Stridor & $2(4.0)$ \\
Hypotension & $4(8.2)$ \\
Angioedema & $4(8.2)$ \\
Shivering & $3(6.1)$ \\
Pruritus & $12(24.5)$ \\
\hline
\end{tabular}

Urticaria, pruritus, angioedema, breathlessness, stridor, shivering, hypotension were prominent symptoms in allergic \& anaphylactic reactions. Fever, chills \& rigors were prominent symptoms in case of febrile non haemolytic transfusion reaction. (Table 3) In our study we found that mean transfusion requirement of AML was more than ALL (p: 0.001) and no relationship between transfusion reaction and unit of transfusion was found (p: 0.123). (Table 4)

Table 4: Distribution of transfusion reaction by unit of transfusion.

\begin{tabular}{lccl}
\hline Required Transfusion & Subjects & $\mathbf{n}(\%)$ & P-value \\
\hline $0-10$ unit & 51 & $10(19.6)$ & \\
$11-20$ unit & 23 & $9(39.13)$ & \\
$21-30$ unit & 6 & $3(50.0)$ & $0.203 \mathrm{~ns}$ \\
$31-40$ unit & 12 & $3(25.0)$ & \\
$41-50$ unit & 3 & $0(0.0)$ & \\
\hline
\end{tabular}

\section{Discussion}

In this study mean age for AML and ALL was 34.44 and 30.42 respectively. Tallman and Juliusson et al. showed much higher mean age for AML. 2,4 The reason for this difference may be due to geographic/ ethnic influence. Gender distribution of subjects showed female sex predominance $(53.1 \%)$ in AML and male sex predominance $(67.7 \%)$ in ALL but Jackson et al and Li et al described acute leukaemia is more common in males at almost every age and gender ration of male to female patients was 1.84:1.5,6 Among total patients transfusion reactions were observed in $26.3 \%$ subjects. These findings are inconsistent with the results of several other studies conducted by Cho $\mathrm{J}$ et al and Bhattacharya et al which may be due to increased transfusion requirement in acute leukaemia patient in contrast to other no $\mathrm{n}$ haematological

Page | 37 patients. ${ }^{7-9}$ In our study we did not found any significant relationship between age and sex with transfusion reaction. Oakley FD et al found that transfusion reaction incidence was same between sexes in adult patients but in paediatric patients (age $<21$ years), showed reactions were more common in males than female. 10 They also reported that incidence of transfusion reaction was more in paediatric patients than adult patients. In another study, Sidhu et al reported among total transfusion reaction $47.8 \%$ were seen in males and $52.1 \%$ in females. ${ }^{11}$ In our study we didn't found any relationship between transfusion reaction with types of platelet and donor. Tormey et al also showed that no relationship exists between transfusion reaction with types of platelet transfusion. 12 During our study period we didn't found any relationship between transfusion reactions with unit of transfusion. In contrast, Chowdhury et al found that there was a strongly positive relations hip between transfusion reaction and unit on transfusion. ${ }^{13}$ Among the transfusion reactions 8 (32\%) were FNHTR, 12 (48\%) were allergic reactions, 4 (16\%) were anaphylactic reactions and $1(4 \%)$ were DHTR. The frequency of FNHTRs to RCC (62.5\%) was significantly higher than those to platelet concentrate $(37.5 \%)$. In contrast, the frequency of allergic reactions to platelet components $(50 \%)$ was significantly higher than RCC (33.33\%) and FFP (16.67\%). However, the nearly $100 \%$ anaphylactic reactions were observed in case of platelet transfusion. Cho $\mathrm{J}$ et al found that FNHTR to RCC, platelet cone, FFP were $0.9 \%, 0.3 \%$ and $0.2 \%$ respectively. 5 Hirayama found that allergic reactions with platelet and $\mathrm{RCC}$ have an incidence rate $3.7 \%$ and $0.15 \%$ respectively. 14 We observed sign symptoms for individual's pattern of transfusion reaction during our study period. Fever, urticaria, pruritus, shivering, angioedema, breathlessness, stridor, hypotension were the most common sign and symptoms reported in transfusion reactions. Allergic transfusion reactions were the commonest acute transfusion reactions reported and comprised $48 \%$ (n:12) of all reactions. Commonest sign and symptoms were urticaria, pruritus and rigors which were treated by simple oral antihistamine. Anaphylactic reactions were found in $16 \%$ (n:4) patients. The most common sign and symptoms were urticaria, breathlessness, angioedema, stridor and hypotension. Sidhu et al found that commonest acute transfusion reactions were allergic which comprised $41.5 \%$ of all reactions, the most common sign and symptoms were rash, 
pruritus and urticaria. ${ }^{11}$ Dyspnoea and hypotension was the prominent symptoms for anaphylactoid reactions. Similarly, Chavan et al found that allergic reactions were commonest and clinical signs and symptoms were chills and rigors and pruritus. ${ }^{15}$ FNHTR were found in $32 \%$ (n:8) patients. The most common sign and symptoms were fever, chills and rigors. Sidhu et al found that $35.1 \%$ patients developed FNHTR and most common sign and symptoms were fever, chills and rigors, hypotension and myalgia. Delayed haemolytic transfusion reactions $4 \%$ (n: 1) developed in one acute leukaemia patients 5 days after transfusion. There was history of yellowish discoloration of skin and urine, fall in posttransfusion haemoglobin. Peripheral blood film shows features of haemolysis and antibody screening was positive after transfusion. Patient did not recover and died later due to disease process. The overall risk estimates of DHTR cited in various studies vary from 0.007 to 0.6907 per 1000 red cell units transfused.16-18 DHTR is difficult to diagnose and most often, it is asymptomatic or may even be similar to the clinical signs and symptoms of the patient so that it remains under diagnosed and underreported.

\section{Conclusion}

Significant number of patients showed different transfusion reaction in acute leukaemia patient. Therefore, acute leukaemia patient should be monitored closely during transfusion for early detection adverse transfusion reaction.

\section{References}

1. Hossain MS, Iqbal MS, Khan MA, Rabbani MG, Khatun H, Munira S, Miah MM, Kabir AL, Islam N, Dipta TF, Rahman F. Diagnosed hematological malignancies in Bangladesh-a retrospective analysis of over 5000 cases from 10 specialized hospitals. BMC cancer. 2014 Dec;14(1):438.

2. Tallman MS. New strategies for the treatment of acute myeloid leukemia including antibodies and other novel agents. ASH Education Program Book. 2005 Jan 1;2005(1):143-150.

3. Leukaemia. In: Provan D, Singer CR, Baglin T, Dokal I. Oxford handbook of clinical haematology. Oxford university press; 2009; p:132.

4. Juliusson G, Antunovic P, Derolf Å, Lehmann S, Möllgård L, Stockelberg D, Tidefelt U, Wahlin A, Höglund M. Age and acute myeloid leukemia: real world data on decision to treat and outcomes from the Swedish Acute Leukemia Registry. Blood. 2009 Apr 30;113(18):4179-4187.
Original Article

5. Jackson N, Menon BS, Zarina W, Zawawi N, Naing NN. Why is acute leukemia more common in males? A possible sex-determined risk linked to the $\mathrm{ABO}$ blood group genes. Annals of hematology. 1999 May 1;78(5):233-236.

6. Li SY, Ye JY, Meng FY, Li CF, Yang M. Clinical characteristics of acute lymphoblastic leukemia in male and female patients: A retrospective analysis of 705 patients. Oncology letters. 2015 Jul 1;10(1):453-458.

7. Cho J, Choi SJ, Kim S, Alghamdi E, Kim HO. Frequency and pattern of noninfectious adverse transfusion reactions at a tertiary care hospital in Korea. Annals of laboratory medicine. 2016 Jan 1;36(1):36-41.

8. Bhattacharya P, Marwaha N, Dhawan HK, Roy P, Sharma RR. Transfusion-related adverse events at the tertiary care center in North India: An institutional hemovigilance effort. Asian journal of transfusion science. $2011 \mathrm{Jul} ; 5(2): 164$.

9. Lopez-Plaza I, Weissfeld J, Triulzi DJ. The cost-effectiveness of reducing donor exposures with single-donor versus pooled random-donor platelets. Transfusion. 1999 Sep;39(9) :925932.

10. Oakley FD, Woods M, Arnold S, Young PP. Transfusion reactions in pediatric compared with adult patients: a look at rate, reaction type, and associated products. Transfusion. 2015 Mar;55(3):563-570.

11. Sidhu M, Meenia R, Yasmeen I, Akhtar N. A study of transfusion related adverse events at a tertiary care centre in North India: an initiative towards hemovigilance. Intern J Adv Med. 2015 Jul;2:206-210.

12. Tormey CA, Sweeney JD, Champion MH, Pisciotto PT, Snyder $\mathrm{EL}, \mathrm{Wu} \mathrm{Y}$. Analysis of transfusion reactions associated with prestorage-pooled platelet components. Transfusion. 2009 Jun;49(6):1242-1247.

13. Chowdhury FS, Biswas J, Siddiqui MA, Hoque MM, Adnan SK. Transfusion reaction among the blood recipient-A study of 120 cases. Journal of Dhaka Medical College. 2008;17(2):67-71.

14. Hirayama F. Current understanding of allergic transfusion reactions: incidence, pathogenesis, laboratory tests, prevention and treatment. British journal of haematology. 2013 Feb;160 (4):434-444.

15. Chavan SK, Patil G, Rajopadhye P. Adverse blood transfusion reactions at tertiary care hospital. Int J Res Med Sci. 2017;4(6):2402-2407.

16. Moore SB, Taswell HF, Pineda AA, Sonnenberg CL. Delayed hemolytic transfusion reactions. Evidence of the need for an improved pretransfusion compatibility test. American journal of clinical pathology. $1980 \mathrm{Jul}$ 1;74(1):94-97.

17. Lichtiger B, Perry-Thornton E. Hemolytic transfusion reactions in oncology patients: experience in a large cancer center. Journal of Clinical Oncology. 1984 May;2(5):438-442.

18. Robillard P, Itaj N, Corriveau P. ABO Incompatibe Transfusions, Acute and Delayed Hemolytic Transfusion Reactions in the Quebec Hemovigilance System-Year 2000. Transfusion. 2002 Sep 1;42. 